\title{
Pitch Angle Regulation of Floating Wind Turbines with Dynamic Uncertainty and External Disturbances
}

\author{
Najah F. Jasim \\ Department of Mechanical Engineering, University of Kerbala \\ Kerbala 56001, Iraq \\ jasimn2@asme.org
}

\begin{abstract}
This paper addresses the problem of pitch angle regulation of floating wind turbines with the presence of dynamic uncertainty and unknown disturbances usually encountered in offshore wind turbines, where two control laws are derived for two different cases to continuously achieve zero pitch angle for the floating turbine. In the first case, the timevarying unknown coefficients that characterize the turbine's dynamics are assumed reasonably bounded by known functions, where robust controller is designed in terms of these known functions to achieve zero pitch angle for the turbine with exponential rate of convergence. While in the second case, the turbine's dynamics are considered to be characterized by unknown coefficients of unknown bounds. In this case, a slidingmode adaptive controller is constructed in terms of estimated values for the unknown coefficients, where these values are continuously updated by adaptive laws associated with the proposed controller to ensure asymptotic convergence to zero for the turbine's pitch angle. Simulations are performed to demonstrate the validity of the proposed controllers to achieve the required regulation objective.
\end{abstract}

\section{INTRODUCTION}

Winds are considered as one of the main resources for the renewable energy that is becoming increasingly important part in electrical power generation. It was pointed out that terrestrial wind power expansion has slowed down due to several problematic issues such as noise generation and the need for large expanses of land for wind farms set up. Therefore, significant attention has been paid for building wind turbines at sea [1].

According to the European Wind Energy Association (EWEA), over $100 \mathrm{GW}$ of offshore wind projects are already in various stages of planning, which has the capability of producing $10 \%$ of the Europe's electricity whilst avoiding 200 million tonnes of $\mathrm{CO}_{2}$ emissions each year [2]. A government report investigated the feasibility of wind energy to provide $20 \%$ of U.S. electricity by 2030 , and this report found that more than $300 \mathrm{GW}$ of wind energy capacity would need to be installed, including $54 \mathrm{GW}$ offshore [3]. On the other hand, a recent survey study of wind behavior over the Arabian Gulf showed the capability of the this Gulf to provide efficient wind resources, and a wind farm is a prospective renewable energy resource in this region [4].

To some extent, a greater power generation can be achieved if the wind turbine is set up far enough from the coastal regions at depths greater than $50 \mathrm{~m}$ where high speed winds are abundant [1]. In such depths, floating structures are used for deploying offshore wind turbines. In this kind of wind turbine, the two problematic issues just mentioned for terrestrial wind turbines can be avoided. As well as these advantages, the effects of fatigue loads are significantly reduced in this kind of turbines which leads to achieving longer life structures. In addition, such floating wind turbines are very suitable for delivering power for oil and gas industries that are usually available offshore. For these and other reasons, floating wind turbines were the subject of many recent researches that have studied offshore wind turbines from different points of view. Some of theses researches concentrated on the concept of offshore floating wind turbines [6], while significant attention has been paid for the turbines modeling objective [5], [7], [8], [9], [10], and other studies aimed to address design issues and economic assessment of this type of wind turbines [11] and the future of offshore wind energy in some interested countries (see e.g. [12]).

In this context, the problem of pitch angle regulation for floating wind turbines is considered in this paper, and our objective is to design control laws to achieve zero pitch angle for a floating wind turbine whose dynamics is characterized by unknown time-varying coefficients and external disturbances induced from hydrodynamic and aerodynamic interactions of the floating turbine with its environment. The main results are first accomplished for a class of general dynamic systems represented by non-homogeneous differential equation with time-varying unknown coefficients and unknown force function, where sufficient conditions are derived for output regulation of such systems. Then the main results are applied to the problem of pitch angle regulation of a floating wind turbine with dynamic uncertainty and external disturbances. 


\section{PROBLEM STATEMENT}

We consider the general dynamic system described by the following non-homogenous differential equation with timevarying coefficients:

$$
\begin{aligned}
& \alpha(t) \ddot{x}+\beta(t) \dot{x}+\gamma(t) x=F(t)+u \\
& y=\dot{x}+\lambda x
\end{aligned}
$$

where $\alpha(t), \beta(t)$ and $\gamma(t)$ are time-varying coefficients, $\mathrm{F}(\mathrm{t})$ is a generalized force function, $u$ is the control input, $x$ is a state variable, $\lambda$ is strictly positive constant and $y$ is the output of the system.

Throughout this paper, we consider the following assumption.

Assumption 1: The states of the system $x$ and $\dot{x}$ are considered to be locally Lipschitz and available for measurement, and the function $\alpha(t)$ is strictly positive, i.e.

$\alpha(t)>0 \quad \forall t \geq 0$.

Concerning the time-varying coefficients $\alpha(t), \beta(t), \gamma(t)$ and the function $\mathrm{F}(\mathrm{t})$, there are two possible cases for the system as shown in the following two assumptions.

Assumption 2: The time-varying coefficients $\alpha(t), \beta(t)$, $\gamma(t)$ and the function $\mathrm{F}(\mathrm{t})$ are considered to be bounded and unknown, but there exist known functions $\alpha^{*}(t), \beta^{*}(t), \gamma^{*}(t)$ and $\rho^{*}(t) \quad$ such that $|\alpha(t)| \leq \alpha^{*}(t), \quad|\beta(t)| \leq \beta^{*}(t)$, $|\gamma(t)| \leq \gamma^{*}(t)$ and $|F(t)| \leq \rho^{*}(t)$.

Assumption 3: The time-varying coefficients $\alpha(t), \beta(t)$, $\gamma(t)$ and the function $\mathrm{F}(\mathrm{t})$ are considered to be bounded, unknown and of unknown bounds.

Remark 1: Since $\alpha(t), \beta(t), \gamma(t)$ and $\mathrm{F}(\mathrm{t})$ are bounded, there exist unknown constants $\bar{\alpha}, \bar{\beta}, \bar{\gamma}$ and $\bar{\rho}$ such that $\bar{\alpha}=\sup _{t \geq 0}|\alpha(t)|, \quad \bar{\beta}=\sup _{t \geq 0}|\beta(t)|, \quad \bar{\gamma}=\sup _{t \geq 0}|\gamma(t)| \quad$ and $\bar{\rho}=\sup _{t \geq 0}|F(t)|$.

The main objective of this paper is to find control laws for achieving zero pitch angle for the floating turbine considering these assumptions.

Defining the error $e=x-x_{d}$ with the desired state $x_{d}(t)=0$, we get $e=x$. Let us define a filtered error as:

$$
s(t)=\left(\frac{d}{d t}+\lambda\right) e(t)
$$

Then the problem of tracking the states $x_{d}(t)=0$ and $\dot{x}_{d}(t)=0$ can be replaced by a first-order stabilization in $s$ [13], which implies output regulation of system (1) because $y=s$.

\section{MAIN RESULTS}

In this section we find sufficient conditions for output regulation of system (1) that satisfies Assumptions 1 and 2 or Assumptions 1 and 3 . This is respectively accomplished throughout the following two theorems.
Theorem 1: Under Assumptions 1 and 2, the dynamic system described by (1) is asymptotically exponentially stable if it satisfies the following control law:

$$
u=\operatorname{sgn}(s)\left\{-\lambda \alpha^{*}|\dot{x}|-\rho^{*}-\beta^{*}|\dot{x}|-\gamma^{*}|x|-s\right\}
$$

Proof: Differentiating the filtered error s and substituting for $e=x$ and $\dot{e}=\dot{x}$, we get

$$
\dot{s}=\ddot{x}+\lambda \dot{x}
$$

From (1), we have

$$
\ddot{x}=\frac{1}{\alpha}\{F(t)+u-\beta(t) \dot{x}-\gamma(t) x\}
$$

Substitution of (5) into (4) yields

$$
\dot{s}=\frac{1}{\alpha}\{F(t)+u-\beta(t) \dot{x}-\gamma(t) x\}+\lambda \dot{x}
$$

Substituting for $\mathrm{u}$ from (3), we find

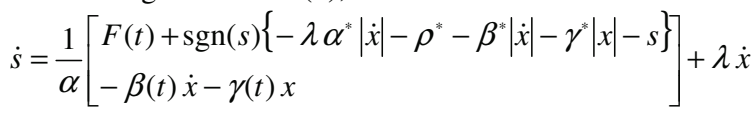

Select the following Lyapunov candidate:

$$
V=\frac{\bar{\alpha}}{2} s^{2}
$$

Differentiating this function and substituting for $\dot{s}$ from (7), we

$$
\begin{aligned}
\dot{V} & =\frac{\bar{\alpha}}{\alpha} s\left[\begin{array}{l}
F(t)+\operatorname{sgn}(s)\left\{-\lambda \alpha^{*}|\dot{x}|-\rho^{*}-\beta^{*}|\dot{x}|-\gamma^{*}|x|-s\right\} \\
-\beta(t) \dot{x}-\gamma(t) x+\lambda \alpha \dot{x}
\end{array}\right] \\
& =\frac{\bar{\alpha}}{\alpha}\left[\begin{array}{l}
s F(t)+|s|\left\{-\lambda \alpha^{*}|\dot{x}|-\rho^{*}-\beta^{*}|\dot{x}|-\gamma^{*}|x|-s\right\} \\
-s \beta(t) \dot{x}-s \gamma(t) x+\lambda s \alpha \dot{x}
\end{array}\right] \\
\text { find } \quad & =\frac{\bar{\alpha}}{\alpha}\left[\begin{array}{l}
s F(t)-\lambda \alpha^{*}|s \dot{x}|-\rho^{*}|s|-\beta^{*}|s \dot{x}| \\
-\gamma^{*}|s x|-s^{2}-s \beta(t) \dot{x}-s \gamma(t) x+\lambda s \alpha \dot{x}
\end{array}\right] \\
& =\frac{\bar{\alpha}}{\alpha}\left[\begin{array}{l}
s F(t)-|s| \rho^{*}-s \beta(t) \dot{x}-\beta^{*}|s \dot{x}| \\
-s \gamma(t) x-\gamma^{*}|s x|+\lambda s \alpha \dot{x}-\lambda \alpha^{*}|s \dot{x}|-s^{2}
\end{array}\right] \\
& \leq \frac{\bar{\alpha}}{\alpha}\left[\begin{array}{l}
s F(t)|-| s\left|\rho^{*}+\right| s \beta(t) \dot{x}\left|-\beta^{*}\right| s \dot{x} \mid \\
+|s \gamma(t) x|-\gamma^{*}|s x|+\lambda|s \alpha \dot{x}|-\lambda \alpha^{*}|s \dot{x}|-s^{2}
\end{array}\right]
\end{aligned}
$$

Since $|s F(t)| \leq|s| \rho^{*},|s \beta(t) \dot{x}| \leq \beta^{*}|s \dot{x}|,|s \gamma(t) x| \leq \gamma^{*}|s x|$ and $\lambda|s \alpha \dot{x}| \leq \lambda \alpha^{*}|s \dot{x}|$, we obtain

$$
\dot{V} \leq-\frac{\bar{\alpha}}{\alpha} s^{2}=-\frac{2}{\alpha} V \leq-\frac{2}{\bar{\alpha}} V
$$

From (8) and (9), we conclude that $V \in L_{\infty}$ and so that $s \in L_{\infty}$. From (9), $\forall t \geq 0$ we have $V(t)-V(0)=\int_{0}^{t} \dot{V} d \tau \leq-\int_{0}^{t} s^{2} d \tau$. So that $\int_{0}^{t} s^{2} d \tau \leq V(0)$ for all $t \geq 0$, which implies $s \in L_{2}$. Since $x$ and $\dot{x}$ are locally Lipschitz, then $\dot{s} \in L_{\infty}$. From $s \in L_{2} \cap L_{\infty}$ and $\dot{s} \in L_{\infty}$, and based on Barbalat's lemma [14], we find that $\lim _{t \rightarrow \infty} s(t)=0$ and so $\lim _{t \rightarrow \infty} y(t)=0$. Therefore, system (1) is asymptotically stable. 
From Lemma 2.1 in [14], we find $V \leq V(0) \exp \left(-\frac{2}{\bar{\alpha}} t\right)$, and so that system (1) is exponentially stable.

$\square$

Theorem 2: Under Assumptions 1 and 3, the dynamic system described by (1) is asymptotically stable if it satisfies the following control and parameter-update laws:

$$
\begin{aligned}
u=\operatorname{sgn}(s)\{ & \{-\lambda \hat{\alpha}|\dot{x}|-\hat{\rho}-\hat{\beta}|\dot{x}|-\hat{\gamma}|x|-s\} \\
& \dot{\hat{\alpha}}=\lambda|s \dot{x}| \\
& \dot{\hat{\beta}}=|s \dot{x}| \\
\dot{\hat{\gamma}} & =|s x| \\
\dot{\hat{\rho}} & =|s|
\end{aligned}
$$

Proof: Substituting (10) into (6), we get

$\dot{s}=\frac{1}{\alpha}\left[F(t)+\operatorname{sgn}(s)\left\{\begin{array}{c}-\lambda \hat{\alpha}|\dot{x}|-\hat{\rho}- \\ \hat{\beta}|\dot{x}|-\hat{\gamma}|x|-s\end{array}\right\}-\beta(t) \dot{x}-\gamma(t) x\right]+\lambda \dot{x}$

Consider the following Lyapunov candidate

$$
V=\frac{1}{2}\left\{\bar{\alpha} s^{2}+\tilde{\alpha}^{2}+\tilde{\beta}^{2}+\tilde{\gamma}^{2}+\tilde{\rho}^{2}\right\}
$$

where $\tilde{(.)}=\hat{(.)}-($.$) .$

Differentiating this function, we obtain

$$
\dot{V}=\bar{\alpha} s \dot{s}+\tilde{\alpha} \dot{\hat{\alpha}}+\tilde{\beta} \dot{\hat{\beta}}+\tilde{\gamma} \dot{\hat{\gamma}}+\tilde{\rho} \dot{\hat{\rho}}
$$

Substituting for $\dot{s}, \dot{\hat{\alpha}}, \dot{\hat{\beta}}, \dot{\hat{\gamma}}$ and $\dot{\hat{\rho}}$, we find

$\dot{V}=\bar{\alpha} s\left(\frac{1}{\alpha}\left[\begin{array}{l}F(t)+\operatorname{sgn}(s)\{-\lambda \hat{\alpha}|\dot{x}|-\hat{\rho}-\hat{\beta}|\dot{x}|-\hat{\gamma}|x|-s\} \\ -\beta(t) \dot{x}-\gamma(t) x\end{array}\right]+\lambda \dot{x}\right)$

$+\lambda \tilde{\alpha}|s \dot{x}|+\tilde{\beta}|s \dot{x}|+\tilde{\gamma}|s x|+\tilde{\rho}|s|$

$=\frac{\bar{\alpha}}{\alpha}\left[\begin{array}{l}s F(t)-\lambda \hat{\alpha}|s \dot{x}|-\hat{\rho}|s|-\hat{\beta}|s \dot{x}|-\hat{\gamma}|s x|-s^{2}- \\ \beta(t) s \dot{x}-\gamma(t) s x+\lambda \alpha s \dot{x}\end{array}\right]$

$+\lambda \tilde{\alpha}|s \dot{x}|+\tilde{\beta}|s \dot{x}|+\tilde{\gamma}|s x|+\tilde{\rho}|s|$

$\dot{V} \leq \frac{\bar{\alpha}}{\alpha}\left[\begin{array}{l}\bar{\rho}|s|-\lambda \hat{\alpha}|s \dot{x}|-\hat{\rho}|s|-\hat{\beta}|s \dot{x}|-\hat{\gamma}|s x|-s^{2}+ \\ \bar{\beta}|s \dot{x}|+\bar{\gamma}|s x|+\lambda \bar{\alpha}|s \dot{x}|\end{array}\right]$

$+\lambda \tilde{\alpha}|s \dot{x}|+\tilde{\beta}|s \dot{x}|+\tilde{\gamma}|s x|+\tilde{\rho}|s|$

$\dot{V}=\frac{\bar{\alpha}}{\alpha}[-\tilde{\rho}|s|-\lambda \tilde{\alpha}|s \dot{x}|-\tilde{\beta}|s \dot{x}|-\tilde{\gamma}|s x|]+\lambda \tilde{\alpha}|s \dot{x}|+$

$\tilde{\beta}|s \dot{x}|+\tilde{\gamma}|s x|+\tilde{\rho}|s|-\frac{\bar{\alpha}}{\alpha} s^{2}$

$\leq-\frac{\bar{\alpha}}{\alpha} s^{2}$
As in the proof of Theorem 1, we can find that $s \in L_{2} \cap L_{\infty}$ and $\dot{s} \in L_{\infty}$, and so that $\lim _{t \rightarrow \infty} s(t)=0$. Therefore, system (1) is asymptotically stable.

\section{SIMULATION}

In this section we apply the main results addressed in Theorems 1 and 2 to a floating wind turbine. The terms required to define translational and rotational motion of a floating wind turbine are shown in Fig. (1).

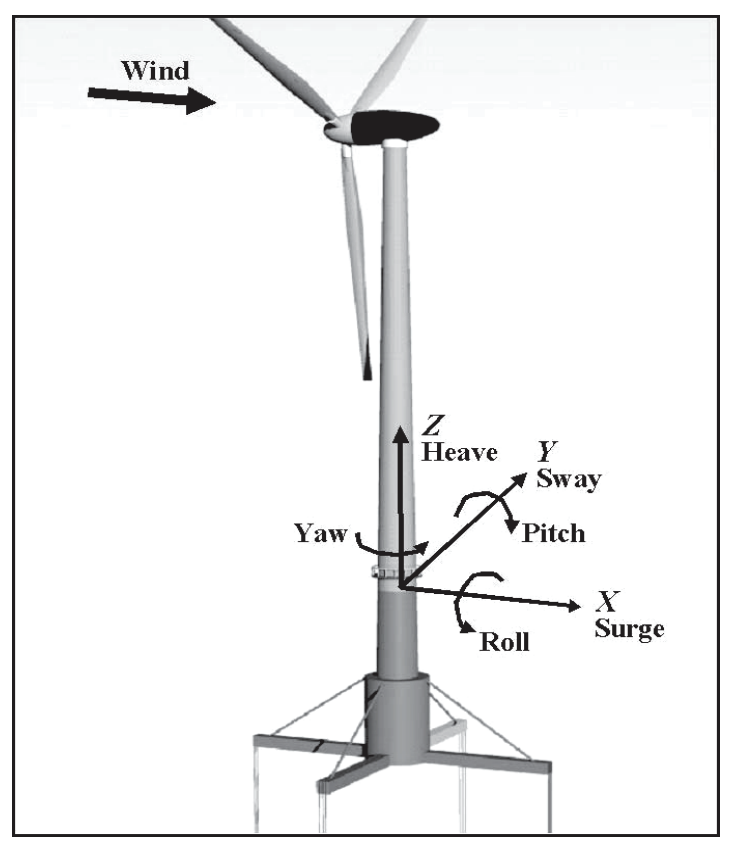

Fig. (1). Coordinate System for Floating Wind Turbine.

After adding a suitable actuator, such as antiroll tanks or any other active control mechanism, for providing the torque required for pitch angle regulation as a control input, the resulting actuator torque can be combined to the pitch dynamics of floating turbine in [5] according to Newton's second law to obtain the following equation of motion:

$$
\begin{aligned}
& \left(I_{\text {Mass }}+A_{\text {Radiation }}\right) \ddot{\xi}+\left(B_{\text {Radiation }}+B_{\text {Viscous }}\right) \dot{\xi}+ \\
& \left(C_{\text {Hydrostatic }}+C_{\text {Lines }}\right) \xi=T(t)+u
\end{aligned}
$$

where $\xi$ is the platform-pitch angle, $\dot{\xi}$ is the platform-pitch rotational velocity, $\ddot{\xi}$ is the platform-pitch rotational acceleration, $I_{\text {Mass }}$ is the pitch inertia associated with wind turbine and barge mass, $A_{\text {Radiation }}$ is the added inertia (added mass) associated with hydrodynamic radiation in pitch, $B_{\text {Radiation }}$ is the damping associated with hydrodynamic radiation in pitch, $B_{V i s c o u s}$ is the linearized damping associated with hydrodynamic viscous drag in pitch, $C_{\text {Hvdrosatic }}$ is the 
hydrostatic restoring in pitch, $C_{\text {Lines }}$ is the linearized hydrostatic restoring in pitch from all mooring lines, $T$ is the aerodynamic rotor-thrust moment and $u$ is the control input.

It is clear that system (18) is a special case of system (1) with $\quad \alpha=\left(I_{\text {Mass }}+A_{\text {Radiation }}\right), \quad \beta=\left(B_{\text {Radiation }}+B_{V \text { iscous }}\right)$, $\gamma=\left(C_{\text {Hydrostatic }}+C_{\text {Lines }}\right), x=\xi$ and $F(t)=T(t)$.

The time-varying coefficients $A_{\text {Radiation }}, B_{\text {Radiation }}, B_{\text {Viscous }}$, $C_{\text {Hydrostatic }}$ and $C_{\text {Lines }}$ characterize complicated aerodynamic and hydrodynamic interactions of the turbine with its environment that is usually uncertain. Therefore, it is very reasonable to consider these coefficients, as well as the torque $T$, to be unknown because of turbine's environment uncertainty and the complicated aerodynamic and hydrodynamic interactions of the turbine with its environment. Then there exist two possible cases; either we have known reasonable upper bounds for these unknown coefficients, or we do not have such bounds, and the turbine's dynamics is of large uncertainty. These two cases are respectively covered by Assumptions 2 and 3 . However, Assumption 1 can be achieved by using accurate devices for measuring the states $\xi$ and $\dot{\xi}$.

We consider a floating turbine of $5300 \mathrm{~kg}$ mass, 2120000 $\mathrm{kg} \cdot \mathrm{m}^{4}$ mass moment of inertia and $40 \mathrm{~m}$ height, subjected to unknown aerodynamic and hydrodynamic effects.

Figure (2) shows the response of system (18) by using the control law (3), while Figure (3) shows the system's response by using controller (10) and parameter-update laws (11)-(14).

The two figures demonstrate the validity of the proposed controllers to achieve the required pitch angle regulation. A comparison between these two figures show the faster convergence of the error signal in the first case where controller (3) is used compared with the second case where controller (10) is employed. This is attributed to the availability of known reasonable bounds to the unknown coefficients in the first case, where these bounding functions were employed in controller (3), in contrast to the second case where such bounding functions are not available, and the control law (10) has used estimated values that are continuously updated using update laws (11)-(14).

\section{CONCLUSION}

Two control laws are designed in this paper for controlling the pitch angle of floating wind turbines characterized by unknown coefficients and external disturbances. Simulations are performed and show the validity of the proposed controllers to achieve zero pitch angle for a floating wind turbine. The first controller is designed for the case where the unknown coefficients and external disturbance are bounded by known functions, while the second controller is developed for the case where such known functions are not available. As shown in the simulation, the first controller achieves a faster convergence compared with the second one, and this is because of the availability of known functions that bound the unknown coefficients and external disturbance in the first case, where these functions are employed in the designed controller, while the second case does not satisfy such assumption. The state of the system in this paper is considered available for measurement, and a future work is recommended to consider the case of unmeasured state, where state observers can be added to ensure the objective of pitch angle regulation.

\section{REFERENCES}

[1] D. Roddier and J. Weinstein, Floating wind turbines. Mechanical Engineering Magazine, ASME, April 2010, 132, pp. 28-32.

[2] European Wind Energy Association, "Oceans of Opportunity, Harnessing Europe's largest domestic energy resource". September 2009.

[3] U.S. Department of Energy, "20\% Wind Energy by 2030: Increasing Wind Energy's Contribution to U.S. Electricity Supply". http://www.20percentwind.org/20p.aspx?page=Report. May 2008.

[4] H. Khonkar, Complete Survey of Wind Behavior over the Arabian Gulf. JKAU: Mar. Sci., Vol. 20, pp: 31-47, 2009.

[5] J.M. Jonkman, Dynamics Modeling and Loads Analysis of an Offshore Floating Wind Turbine. National Renewable Energy Laboratory, Technical Report NREL/TP-500-41958, November 2007.

[6] P. D. Sclavounos, P. D., E. N. Wayman, S. Butterfield, J. Jonkman and W. Musial, Floating Wind Turbine Concepts. European Wind Energy Association Conference (EWEC), Athens, Greece, 27 February - 2 March, 2006.

[7] E. N. Wayman, P. D. Sclavounos, S. Butterfield, J. Jonkman and W. Musial, Coupled Dynamic Modeling of Floating Wind Turbine Systems. Offshore Technology Conference (OTC), Houston, Texas, May 1-4, 2006.

[8] J. E. Withee and P. D. Sclavounos, Fully Coupled Dynamic Analysis of a Floating Wind Turbine System. 8th World Renewable Energy Congress 2004.

[9] T. Zambrano, T. MacCready and T. Kiceniuk, D. G. Roddier and C. A. Cermelli, Dynamic Modeling of Deepwater Offshore Wind Turbine Structures in Gulf of Mexico Storm Conditions. Proceedings of OMAE 2006, 25th International Conference on Offshore Mechanics and Arctic Engineering, June 4-9, 2006, Hamburg, Germany

[10] H. Moll, F. Vorpahl and H. Busmann, Dynamics of Support Structures for Offshore Wind Turbines in Fully-coupled Simulations - Influence of Water Added Mass on Jacket Mode Shapes, Natural Frequencies and Loads. Proceedings of the European Wind Energy Conference and Exhibition 2010

[11] P. D. Sclavounos, C. Tracy and S. Lee, Floating Offshore Wind Turbines: Responses in a Seastate, Pareto Optimal Designs and Economic Assessment. OMAE 2006 Conference, Lisbon, Portugal.

[12] W. Musial S. Butterfield, Future for Offshore Wind Energy in the United States. Energy Ocean 2004 Palm Beach, Florida June 28-29, 2004

[13] J.-J. E. Slotine and W. Li, Applied nonlinear control. Englewood Cliffs, NJ: Prentice-Hall, 1991

[14] J. T. Spooner, M. Maggiore, R. Ordóňez, and K. M. Passino, Stable adaptive control and estimation for nonlinear systems. John Wiley \& Sons, Inc., 2002 

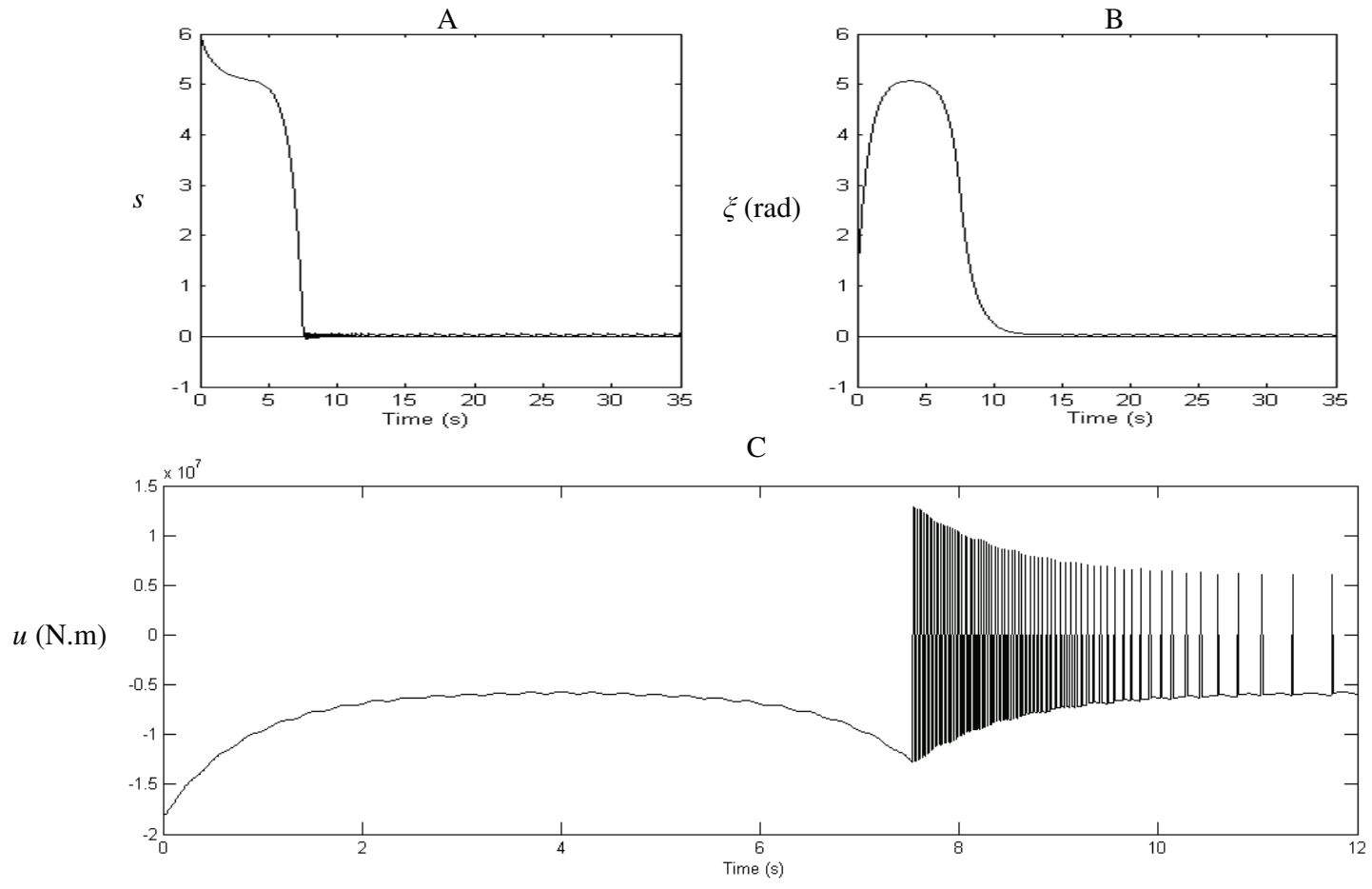

Fig. (2). A- Filtered error response via control law (3). B- Pitch angle regulation via control law (3). C- Control variable $u$ described by (3).
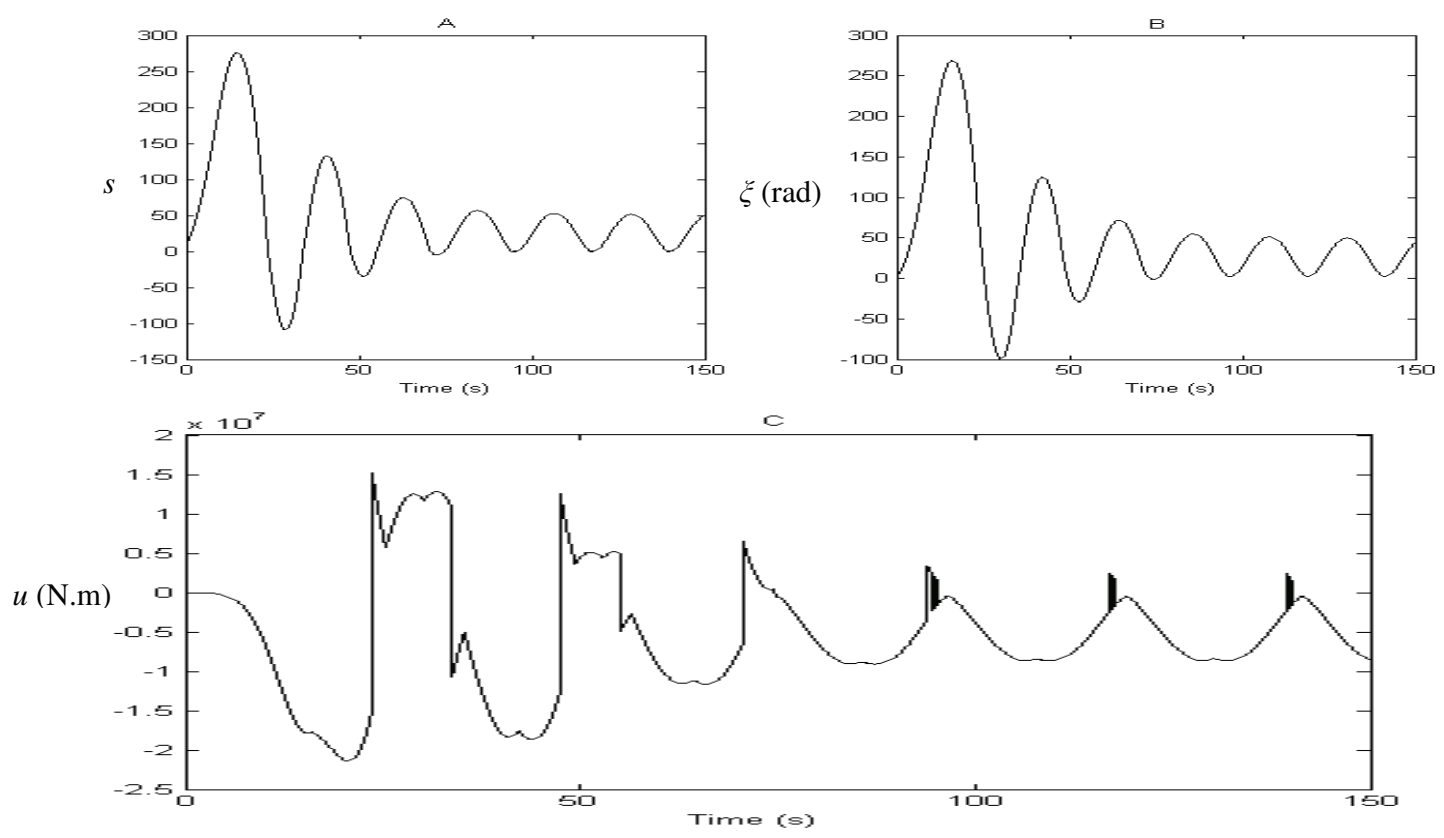

Fig. (3). A- Filtered error response via control law (10). B- Pitch angle regulation via control law (10). C- Control variable $u$ described by (10). 